\title{
¿S Research Square

\section{Mapping implementation climate for integrating evidence-based task-strengthening strategies for hypertension control within HIV clinics in Nigeria}

Juliet Iwelunmor ( $\square$ juliet.iwelunmor@slu.edu )

Saint Louis University College for Public Health and Social Justice https://orcid.org/0000-0003-13765443

\section{Oliver Ezechi}

Nigerian Institute of Medical Research

Chisom Obiezu-Umeh

Saint Louis University College for Public Health and Social Justice

\section{David Oladele}

Nigerian Institute of Medical Research

\section{Ucheoma Nwaozuru}

Saint Louis University College for Public Health and Social Justice

\section{Angela Aifah}

New York University School of Medicine

Joyce Gyamfi

New York University School of Medicine

Titilola Gbajabiamila

Nigerian Institute of Medical Research

Adesola Z. Musa

Nigerian Institute of Medical Research

\section{Deborah Onakomaiya}

New York University School of Medicine

\section{Ashlin Rakhra}

New York University School of Medicine

Hu Jiyuan

New York University School of Medicine

\section{Oluwatosin Odubela}

Nigerian Institute of Medical Research

\section{Ifeoma Idigbe}

Nigerian Institute of Medical Research

\section{Bamidele 0. Tayo}

Loyola University Chicago Stritch School of Medicine 


\section{Gbenga Ogedegbe}

New York University School of Medicine

\section{Research}

Keywords: Hypertension control, HIV clinics, Implementation Climate, Nigeria

Posted Date: March 31st, 2021

DOI: https://doi.org/10.21203/rs.3.rs-339833/v1

License: (c) (1) This work is licensed under a Creative Commons Attribution 4.0 International License. Read Full License 


\section{Abstract}

Background: Evidence-based task-strengthening strategies for hypertension (HTN) control (TASSH) are not readily available for patients living with HIV in sub-Saharan Africa where the dual burden of HTN and HIV remains high. We are conducting a cluster randomized controlled trial comparing the effectiveness of practice facilitation versus a self-directed control (i.e., receipt of TASSH with no practice facilitation) in reducing blood pressure and increasing the adoption of task-strengthening strategies for HTN control within HIV clinics in Nigeria. Prior to implementing the trial, we conducted formative research to identify factors that may influence the integration of TASSH within HIV clinics in Nigeria.

Methods: This study was conducted with purposively selected healthcare providers at 29 HIV clinics, followed by a one-day stakeholder meeting with 19 representatives of HIV clinics. We collected quantitative practice assessment data using two instruments: a) an adapted Service Availability and Readiness Assessment (SARA) tool to assess the capacity of the clinic to manage NCDs and b) Implementation Climate Scale to assess the degree to which there is a strategic organizational climate supportive of the evidence-based practice implementation. The quantitative data were analyzed using descriptive statistics and measures of scale reliability. We also used the Consolidated Framework for Implementation Research (CFIR), to thematically analyze qualitative data generated and relevant to the aims of this study.

\section{Results:}

Across the 29 clinics surveyed, the focus on TASSH (mean=1.77 (SD=0.59)) and educational support (mean=1.32 ( $(\mathrm{SD}=0.68)$ ) subscales demonstrated the highest mean score, with good-excellent internal consistency reliability (Cronbach's alphas ranging from 0.84 to 0.96 ). Within the five CFIR domains explored, the major facilitators of the intervention included relative advantage of TASSH compared to current practice, compatibility with clinic organizational structures, support of patients' needs, and intervention alignment with national guidelines. Barriers included perceived complexity of TASSH, weak referral network and patient tracking mechanism within the clinics, and limited resources and diagnostic equipment for HTN.

\section{Conclusion:}

Optimizing healthcare workers' implementation of evidence-based TASSH within HIV clinics requires attention to both the implementation climate and contextual factors likely to influence adoption and longterm sustainability. These findings have implications for the development of effective practice facilitation strategies to further improve the delivery and integration of TASSH within HIV clinics in Nigeria.

\section{Contributions To The Literature}

- Investigates the facilitators and barriers to the implementation of an evidence-based taskstrengthening strategies for hypertension control within HIV clinics in Nigeria. 
- Identifies strengths and opportunities to leverage within HIV clinics and some challenges to address to optimize for the implementation of task-strengthening strategies for hypertension control within HIV clinics in Nigeria.

- Findings will inform a practice facilitation strategy to enhance the implementation and integration of evidence-based task-strengthening strategies for hypertension control within HIV clinics in Nigeria.

\section{Introduction}

Hypertension (HTN) remains a key risk factor for cardiovascular diseases (CVD), particularly in subSaharan African countries currently undergoing the epidemiologic transition of mortality from infectious disease to non-communicable disease (NCD) [1, 2]. People living with HIV (PLHIV) are at an increased risk for CVD due to an increased prevalence of traditional risk and non-traditional risk factors (i.e.

inflammation) as well as the effects of antiretroviral drugs [3]. Evidence-based strategies for HTN control (i.e., World Health Organization's Package of Essential NCD Interventions for Primary Health Care in lowresource settings (WHO-PEN)) are important but underutilized for hypertension treatment and control among PLHIV [4, 5]. Additionally, the shortage of healthcare workers limits the effective reduction of HTNrelated morbidity and mortality rates among PLHIV [6]. Task-strengthening strategies, particularly engaging health care workers such as nurses to deliver the WHO-PEN package may mitigate the optimal HTN control among PLHIV $[7,8]$. However, integration of these services within HIV clinics in SSA is nonexistent.

One factor that may impact the integration, implementation, adoption and sustainability of evidencebased task-strengthening strategies for HTN control is the implementation climate [9]. Defined here as shared perceptions on the importance of evidence-based practice implementation within an organization, Erhart et al., (2014) suggest that implementation climate creates a fertile organizational context for putting an evidence-based intervention into practice in an organization [10]. The concept has been applied to explore, for example, individual and organizational factors that influence the implementation of interventions for children with autism [11], to optimize clinician's implementation of evidence-based practice in behavioral health organizations [12], as well as to assess predictors of engagement in a community-based learning collaborative [13].

Although implementation climate has a robust theoretical foundation [12, 14]; few empirical studies have explored its influence with implementing evidence-based practices

in sub-Saharan Africa. As a result, closing the gap between research and practice is stymied by the paucity of research on the implementation climate necessary for integrating evidence-based HTN interventions into routine care for PLHIV. Williams and Erhart (2018) suggest that this is an important gap, because once an implementation climate is established, it may potentially have an on-going, longterm influence on the behavior of health care workers, including those who subsequently enter the organizations [12]. There is evidence that practice facilitation, which provides external expertise on practice redesign and promotes a tailored approach to implementing systems changes to improve patient 
outcomes, may mitigate barriers to implementation climate [12]. It may also generate effective implementation strategies that include health care workers' perceptions and their use of evidence-based practices including what is expected, supported, and rewarded by their organization [10].

We are conducting a cluster randomized controlled trial that is comparing whether practice facilitation (PF) will reduce blood pressure among PLHIV and increase the adoption and sustainability of taskstrengthening strategies for hypertension control. The control sites will receive self-directed conditions (i.e., receipt of the information on TASSH without PF), while the intervention sites will receive TASSH plus practice facilitation. Prior to implementation and to maximize the opportunity for success with the integration of evidence-based task-strengthening strategies for hypertension control within HIV clinics in Nigeria, we sought to explore key stakeholders' current perceptions of barriers and facilitators to the implementation of TASSH in HIV clinics and organizational readiness for change. Findings will inform the adaptation strategies needed to tailor TASSH implementation to the local context.

\section{Methods}

\section{Study design and setting}

The study was conducted in Lagos, the largest city in Nigeria, and led by the Nigerian Institute of Medical of Research (NIMR). We conducted a cross-sectional study design employing a sequential (Quan $\rightarrow$ Qual) mixed-methods approach - a quantitative first step using structured questionnaires followed by a qualitative approach using stakeholder meetings. Data were collected between October 2017 and January 2018. Ethical approval for this study was obtained from the NIMR Institutional Review Board.

\section{Identification of HIV Clinics and Key stakeholders}

Quantitative data were collected using a modified practice assessment survey to examine the practice capacity of 29 geographically distinct HIV clinics across 20 different Local Government Areas in Lagos State. The 29 clinics were purposively selected based on the provision of comprehensive ART services at the clinic sites, patient load including private, public/government, faith-based organizations, and primary facility level. For the qualitative aspect, we convened a stakeholder's meeting of 19 representatives of HIV and non-communicable disease organizations from Lagos State Ministry of Health, Lagos State Primary Health Care Development Agency, Lagos State AIDS Control Agency, and the Nigerian Institute of Medical research. Stakeholders were purposively recruited to represent individuals who were either directly involved as a participating clinician in the management of NCDs and HIV or involved directly or indirectly at a policy or strategic level.

\section{Quantitative data collection}

We collected quantitative practice assessment data using two instruments: a) an adapted Service Availability and Readiness Assessment (SARA) tool to assess the capacity of the clinic to manage NCDs 
and b) Implementation climate scale to assess the degree to which an organization is supportive of evidence-based practice implementation.

Service Availability and Readiness Assessment: The tool collected data on:1) Demographics (provider's age, educational level, nature of the facility, years of experience in HIV care), 2) Organizational characteristics (proportion of HIV patients with a diagnosis of HTN, availability of tools for diagnosis and treatment of hypertension, financing mechanism), 3) Healthcare provider characteristics (case diagnosis, treatment, referral, and clinic follow-up patterns), and 4) Patient characteristics (access to information related to hypertension management and lifestyle behaviors).

Implementation Climate Scale: Developed by Ehrhart, M.G., Aarons, G.A et al.[10], this modified 14-item scale measures the degree to which evidence-based practices for hypertension management are implemented within the selected HIV clinics. The scale assessed the following: 1) organization focus on EBP for hypertension treatment; 2) educational Support for EBP; 3) recognition for EBP 4) reward for EBP and; 5) openness to implementation. One scale (the selection for EBP) was excluded as it was irrelevant to this context. The objective response options are "2" yes, "1" no or " 0 " don't know, with higher scores indicating a more positive implementation climate.

\section{Qualitative data collection}

We conducted a one-day stakeholder meeting, hosted by NIMR with the key stakeholders mentioned above. The meeting discussion covered: participant's understanding of the purpose of TASSH and its value in the HIV clinics; their perception of its adaptability and impact on nurses' work; and suggestions on how they can become engaged in facilitating the adoption of TASSH within the HIV clinics. The meeting lasted for two hours and was digitally recorded. We used the Consolidated Framework for Implementation Research (CFIR), to thematically analyze data generated from the stakeholder meeting. CFIR provides a pragmatic structure for exploring barriers and facilitators to the implementation of an evidence-based intervention in health systems based on five domains (intervention characteristics, outer setting, inner settings, characteristics of individuals, and the implementation process) $[15,16]$.

\section{Data Analysis}

A sequential analytical strategy was applied which involved a quantitative analysis followed by a qualitative analysis. Descriptive statistics (mean and standard deviation, percent or median, and interquartile range (IQR)) were used to summarize responses to the survey items using SPSS (Statistical Package for Social Sciences) software version 25. The implementation climate scale measures were examined for internal consistency reliability, and Cronbach alphas were calculated for each subscale. The data from the stakeholder's meeting (in an audio-recording device) were first transcribed and then analyzed using content analysis. Using a deductive approach, the 5 CFIR domains and 39 constructs were used to identify prior codes to develop the initial codebook. Prior to coding, the research team reviewed and familiarized themselves with the CFIR coding definitions suggested by Damschroder et al. (2009) [17]. Two coders ( $\mathrm{CO}$ and UN) double-coded the transcripts and identified emerging themes related 
to each CFIR sub-construct and the larger CFIR domain. Discrepancies, such as distinguishing the definitions for intervention complexity and intervention compatibility, between the two coders were resolved by open discussions with a third researcher. The larger research team (JI, CO, UN, DO, AA, and O0) discussed the preliminary themes to reach a consensus on final deductive themes and triangulated responses from the stakeholder's meeting with survey data. Triangulation was achieved by collecting data from different sources (stakeholders meeting and qualitative survey) to facilitate a deeper understanding of barriers, and facilitators influencing effective implementation from multiple perspectives. We applied Guba's qualitative trustworthiness criteria to ensure that the data was collected, analyzed, and interpreted accurately [18]. An audit trail of the coding process was also maintained.

\section{Results}

\section{Study Participants}

The HIV clinics that participated in the survey represented 20 local government areas in Lagos, creating variability in the organizational characteristics and context. The vast majority of the health facilities surveyed were public, government-operated clinics $(97 \%, n=28 / 29)$. Among the health facility representative surveyed, $62 \%(n=18 / 29)$ of the respondents were females, the median age of 40 years (IQR: $37-45$ years), and had worked at the current institution for less than 10 years $(n=21 / 29)$. On average, clinic providers care for 394 HIV patients per month. The number of hypertensives, HIV patients are seen at the facility ranged from 5 to 73 patients per month. Among the participants who attended the stakeholders meeting, 8 of the participants were health providers (noted as HP in the quotes), such as physicians and nurses, within HIV clinics, whereas 11 of them were key informants (noted as SH in the quotes) from the Ministry of Health at the local government level and/or national level.

\section{Implementation Climate for TASSH}

As shown in Table 1, most of the scale measures had good-excellent internal reliability in these samples, with Cronbach's alpha for most subscales ranging from 0.84 to 0.96 . The total average score on the organizational context scale was $1.23(\mathrm{SD}=0.46)$. The focus on TASSH subscale $(1.77(S D=0.59))$ and educational support $(1.31(\mathrm{SD}=0.68)$ ) subscale demonstrated the highest mean score. The selection for openness to TASSH and recognition for utilizing TASSH subscales were rated slightly lower at 1.15 $(S D=0.73)$ and $1.14(S D=0.79)$, respectively. The rewards for utilizing TASSH subscale were rated the lowest at $0.73(S D=0.48)$, which indicates that financial incentives for TASSH use may be uncommon within the clinics.

\section{Responses mapped to CFIR}

Of the 39 CFIR constructs assessed, 11 of the constructs emerged either as barriers or facilitators of integrating task-shifting strategies for HTN control within HIV clinics. Figure 1 provides a visual representation of key areas where additional effort or support could be important for successful implementation. Some constructs were found to be more dominant than others based on the degree of 
importance articulated by the stakeholders and clinic representatives. The CFIR constructs were more concentrated in the inner settings domain compared to the other 4 domains. The relevant constructs within each domain are reported below, including illustrative quotations [table 2].

\section{CFIR-based facilitators with integrating TASSH within HIV Clinics}

\section{The relative advantage of TASSH}

By delegating certain tasks to less specialized health cadres (i.e. nurses), the majority of the stakeholders perceived this model as a solution to make more efficient use of the existing workforce and reduce the workload of overburdened healthcare professionals.

A few health providers highlighted that the rational distribution of the clinical duties among cadres will allow the more specialized healthcare workers to focus solely on clinical tasks and procedures restricted to higher-level cadres. One of the healthcare providers' notes:

"key aspect in the management of hypertension is diet, physical activity, taking appropriate medication and stress management which don't require the attention of a doctor to achieve...if this task is shifted to them, the Doctors could focus on more complex tasks that require co-morbid management." (HP 1)

Nonetheless, $79 \%$ ( $n=23 / 29)$ of the HIV clinic representatives surveyed reported that doctors were the sole providers of lifestyle-related information for HTN management such as prompting heart-healthy diet, low sodium intake, physical activity, and eliminating tobacco and caffeine intake.

Another advantage mentioned was the fact that integrating TASSH into routine care for PLHIV will offer patients the ability to access care in the same clinic or location, which may reduce clinic wait times and stigma associated with care-seeking among PLHIV in some settings.

"Integrating HIV and Hypertension care would reduce the stigma; it would reduce waiting time and there would be more Doctors available to attend to the patient." (SH 1)

\section{Compatibility with existing workflows and processes}

Similar to the network and communications construct, several stakeholders suggested the integration of the existing community health extension workers (CHEWs) into the referral systems, as this may strengthen the compatibility of TASSH intervention within the HIV clinics.

"Community Health Extension Workers (CHEWs) could be useful in the tracking of patients, given their close understanding of the community and direct connections with the community members, which could help take some burden off the Nurses. They could serve as a liaison to provide a comprehensive system which starts from the home down to the health system to further strengthen the referral network for the intervention." (SH3) 
Within the HIV clinics, less than a quarter of the clinic representatives surveyed indicated that CHEWs and other community health officers are responsible for identifying $(21 \%, n=6 / 29)$ and referring $(14 \%, n=4 / 29)$ HIV patients who are hypertensive, suggesting significant gaps in the continuum of care for HTN.

\section{Access to knowledge and information}

Access to training opportunities and educational resources, about TASSH and how to incorporate it into the existing organizational processes were perceived as important facilitators for promoting initial uptake and sustained use of new processes within the facilities. This is particularly important as only $52 \%$ $(n=15 / 29)$ of health facilities surveyed reported that there were training materials, journals, and other educational resources available for evidence-based practice for hypertension management within the HIV clinics. Similarly, 52\% ( $n=15 / 29)$ indicated that their facilities provide specific conferences, workshops, or seminars on evidence-based practices. To illustrate the importance of training, one of the stakeholders provided an example where initial training of staff to implement a new process within the clinic gave them a sense of empowerment, thereby increasing their self-efficacy to carry out the given process.

"The training allowed them to do things they never imagined they could do and it was realized that research training was important to them as it enhanced implementation across the system and made the Nurses feel empowered" (SH12)

Needs and resources of those served by the intervention

Patient needs was a major implementation driver. Majority of the stakeholders supported the goal of the TASSH intervention because they felt that it addressed their long-standing concerns about the growing burden of NCDs among HIV patients at their facilities and how to integrate HTN care into HIV clinics, as described by a stakeholder that,

"patients are no longer dying of AIDS at the rapid rate they used to, they are living longer; thus, NCD's such as cardiovascular diseases and hypertension are now becoming more problematic; the question now is, how can we integrate the treatment of NCD's within an already existing HIV care" (SH12)

\section{$\underline{\text { Incentives }}$}

Stakeholders expressed that health workers at the facilities would expect some form of non-monetary incentives that are both intrinsically and extrinsically motivating to enhance the productivity of health workers with new or additional responsibilities. Some of the suggestions to accommodate the need to incentivize the health workers included social recognition, an increase in professional status, and/or competencies backed by certification.

"there is a need to institutionalize the initiative of providing incentives in form of professional development, as Health workers want something to showcase as part of their achievements in their CVs [Curriculum Vitae]" (SH7) 
Furthermore, monetary incentives were perceived by the stakeholders as an unsustainable form of compensation for managing health worker's productivity. All the clinic representatives surveyed $(n=29 / 29)$ indicated that providers at their health facility neither receive financial incentives nor commissions to use evidence-based practices for the management of diseases.

\section{Knowledge and beliefs}

Despite the potential of strain that could result from adding-on a new responsibility, as highlighted by one of the stakeholders, most of the health providers articulated their belief that the benefits of implementing TASSH within the HIV clinics outweigh any disadvantages. Of the HIV clinic representatives surveyed, $90 \%(n=26 / 29)$ of respondents indicated that evidenced-based practices for hypertension treatment are important to the providers in their health facilities.

\section{Planning}

To avoid the potential of overburdening the health workers, a stakeholder felt that the training required to enable a cadre to take on the new responsibilities should accommodate shift preferences and minimize scheduling conflicts. To do this, it was suggested that the research team consider on-site training at the health facilities as compared to offsite training in the different locations. Other suggestions that emerged within this theme was the need to implement simplified data collection tools to ease adaptation to routine data collection at the clinic and accommodate various reading comprehension levels among the participants. It was also important to the stakeholders that the programs' goals align with the existing national guidelines for NCDs and clinic activities.

"it is imperative to conform, from the beginning of the project to the existing National guidelines for NCDs so that whatever is used will be in-line with the priority actions in the guideline" (SH12)

\section{Engagement}

Critical to the successful development and implementation of the tailored TASSH intervention, the health providers emphasized the importance of engaging and retaining key individuals, particularly those identified as change agents or program champions within the HIV clinics, needed to provide governance. Facilitation of structured training, refresher courses, and adequate supportive supervision were regarded as essential implementation enablers and core leadership qualities needed in the change process.

Furthermore, to sustain the intervention, stakeholders recommended continuous stakeholder engagement at every level of implementation and community awareness of the program.

\section{CFIR-based barriers with integrating TASSH within HIV clinics}

\section{Complexity of TASSH}

Discussion on the perceived difficulties of implementing the TASSH intervention within HIV clinics centered on challenges with dynamic role boundaries. Many cited the potential for disagreements and 
conflicts over roles and role boundaries among the cadres of health workers. The issues of power and authority were commonly cited as important factors that may influence relationships and patterns of collaboration among the health care teams. In this regard, some of the stakeholders emphasized the need to first understand the scope of practice for the lower-cadre healthcare workers at various facility levels, identify overlapping responsibilities, and then define the roles of the healthcare workers.

"We need to look at how services are delivered by these health workers at the primary and secondary care level especially as most patients in HIV care go to the primary care level." (SH7)

"persons involved need to know their limitations particularly as Nurses and CHEW are on the same salary scale and have different entry qualifications... We need to clearly define tasks that would be reallocated and let limitations be clearly identified" (SH 5)

\section{Available resources and support}

Generally, it was found that primary health facilities were supported by the state ministry of health to ensure the availability of basic supplies, diagnostic equipment and first-line drug regimens related to HIV and hypertension management, but this level of support was not uniform across the different clinics, particularly at lower-level facilities. For instance, only 59\% $(n=17 / 29)$ of the HIV clinics surveyed reported that antihypertensive drugs were often readily available at their clinics. This generated some concerns among the stakeholders as they emphatically stated that this deficiency may stifle the implementation of the TASSH intervention within HIV clinics, as resource availability was viewed to be critical in determining the level of staffs' readiness.

"there is a gap, a big discrepancy within the system. Within a small locality, there may be a flagship clinic that is better equipped than a general hospital and within that small locality there may be a primary health clinic that has just one Health care worker (HCW) catering to a lot of people" (SH6)

"A select number of flagship clinics are well stocked with drugs and equipment compared to the others. Some buoyant LGA's have large laboratory which serves the PHC, while at some PHCs, there may have no drugs for the treatment of Hypertension" (SH5)

\section{Networks and communication}

In relation to referral channels and communications, most of the participants emphasized the need to strengthen referral networks for HTN management in order to support the decentralization of service delivery in the context of the task-shifting approach. This was seen to be particularly useful in the event that the health worker is faced with patient needs beyond their level of competence which may require higher-level consultation or referral. It was also noted that there were "no proper patient tracking mechanisms" (SH7) in place for intra-clinic referrals and referrals between clinics and hospitals.

\section{External Policies}


In the context of national policies, the national Multi-Sectoral Action Plan for the

Prevention and Control of Non-Communicable Diseases (2019-2025) from the Federal Ministry of Health in Nigeria identifies task-shifting as a priority action for NCD

management at all levels of care. In addition, we found that there is an existing task-shifting and tasksharing national policy for essential health care services in Nigeria published in 2014. Although these policies exist, stakeholders highlighted that the policies and guidelines are not implemented at the clinics. Additionally, a stakeholder added that the task-shifting and task-sharing national policy lacked specific actions for minimizing the workload of health workers in the clinics.

"The issue we have now is that there is no policy action that says we can only have a health worker take care of 50 people max, and beyond 50 people no more" (SH12)

\section{Discussion}

This study aimed to examine stakeholder's perceptions of the implementation climate and contextual factors necessary for integrating evidence-based task-strengthening strategies for hypertension control within HIV clinics in Nigeria. Using CFIR allowed for a more robust formative assessment that led to the identification of adaptation strategies needed to tailor TASSH implementation to the local context. Understanding the implementation climate from key stakeholders has been identified as a fundamental component for intervention adoption and sustainment, as engaged stakeholders are associated with higher overall positive attitudes towards the adoption of evidence-based practices in their organizations $[19,20]$. The results of the implementation climate subscale on the focus of TASSH and the provision of educational support were high indicating a supportive climate for integrating the intervention within HIV clinics in Nigeria. When key stakeholders' perceptions of TASSH implementation climate, vis a vis a focus on the intervention and provision of educational resources, is high it signals a shared belief that TASSH may become a lasting priority at the HIV clinics rather than a passing trend.

Indeed, participants were of the opinion that TASSH should be given top priority with managing and controlling high blood pressure rates among people living with HIV. In addition, the provision of in-service training alongside training materials, workshops, and seminars on TASSH were considered to be important for shaping health care workers' implementation behavior, ultimately enhancing blood pressure outcomes for people living with HIV. On the other hand, the results of the rewards subscales demonstrated that incentives such as monetary rewards were less likely to affect the implementation of TASSH within HIV clinics. Rather, support and recognitions beyond financial rewards are more likely to grow intrinsic motivation to learn about and adopt TASSH. Taken together, findings illustrate how organizational leaders at HIV clinics can align organizational policies, procedures, and practices as well as provide educational support to create an implementation climate that shifts health care workers' attitudes and motivations towards the effective use of TASSH in practice. The presence of committed and well-informed organizational leadership and program champions has been identified as key 
facilitators in other studies that can mitigate organization barriers by maintaining strategic direction and ensure that organizational resources are directed to support intervention adoption [21-23].

In addition to the implementation climate, the results of this study indicate that there are important contextual factors within the organizations that may facilitate or hinder the integration of TASSH within HIV clinics in Nigeria. This finding is corroborated by previous studies that emphasize the importance of the fit between the intervention model and organizational characteristics that are fundamental for the successful implementation of EBPs $[15,24]$. Specifically, study participants stated that the compatibility of TASSH with existing workflows and access to educational support may be hindered by limited resources for basic supplies, diagnostic equipment, and first-line drug regimens related to HIV and hypertension management. In addition to the weak referral networks, which underscores the mutability of inner context factors and their potential role in shaping TASSH integration within HIV clinics. Overall, the findings underline the potential role of organizational resources and workforce processes in the successful integration of TASSH within participating study sites. Therefore, implementation of TASSH within HIV clinics in Nigeria should include expert consultation at implementing clinics to enhance intervention-settings fit.

Likewise, while monetary incentives were described as least beneficial with TASSH implementation climate, some stakeholders believed extrinsic incentives such as organizational recognitions, increase in professional status, and/or competencies backed by certification not only fosters more commitment but gives health care workers a sense of ownership with TASSH implementation. The relative advantage of TASSH particularly with reducing workloads of overburdened health care workers, reducing clinic wait times, and increasing access to care alongside the potential complexity of TASSH underscores how intervention characteristics may exert positive, negative, or potentially neutral influence on the implementation and integration of TASSH within HIV clinics. More research is needed to understand this relationship and to test the impact of healthcare provider-focused interventions on the implementation climate and long-term sustainment of the intervention. However, planning and engagement with key stakeholders throughout the process of integrating TASSH within HIV clinics would enhance successful implementation.

\section{Implications for practice facilitation and research}

The current study contributes to the existing literature by examining stakeholders' perspectives of the implementation process and generates new evidence by understanding the implementation climate for the integration of task-strengthening strategies to improve HTN control among people living with HIV. Future research is needed to expand our results on how organizational climate drives practice facilitation for evidence-based practices in Nigeria. Findings from this study also provide important insights for developing implementation strategies to develop a positive and strong implementation climate within HIV clinics where TASSH will be implemented. This study underscores the importance of facilitators training and striving for context fit (e.g. leadership support and availability of resources) to facilitate climates conducive for intervention implementation. 


\section{Study strengths and limitations}

The results of this study should be interpreted considering some limitations. First, the sample is limited to the 29 participating organizations and their staff. As such, our findings may not be generalizable. Nonetheless, the organizations selected for the study are within the NIMR network, where the randomized controlled trial will be conducted. Lessons learned from the proposed study could inform implementation in other locations across Nigeria and are transferable to future practice-based interventions. Second, the study relied on self-reported assessments from the study participants, and therefore depends on the stakeholders' perceptions and relationships with the organizations. While the self-reports by stakeholders is an optimal way to measure their perceptions of the organizational climate, it may not necessarily provide an objective evaluation of the organization's realities or practice capacities of the health centers. Third, given the cross-sectional data collection approach utilized for this study, it precludes our ability to make causal statements about the organizational climate that may influence attitudes towards TASSH over time [25]. Future studies should address this limitation by observing trends in perceptions over time, to provide a more robust understanding of the implementation climates at the organizations. This can inform the adoption of implementation strategies to best fit the dominant implementation climate.

Despite these limitations, there are several strengths to this study. First, this study utilized both quantitative and qualitative data to improve our understanding of the implementation climate for the integration of TASSH in HIV clinics. The qualitative data not only corroborated the quantitative findings but added more nuanced information on the implementation climate for TASSH integration [26]. Previous implementation science studies have highlighted the importance of utilizing a mixed-methods approach to achieve a holistic understanding of the multiple factors that implementation [26-28]. Second, this study included stakeholders from multiple organizational levels including health providers policymakers, program coordinators, and program supervisors. Third, to the best of our knowledge, this is the first study to examine the implementation climate for integrating evidence-based task-strengthening strategies for hypertension control within HIV clinics in Nigeria. The knowledge gained from this study will strengthen the implementation of evidence-based practices for HTN control in HIV clinics by offering insights into the barriers and facilitators of successful integration of task-strengthening strategies for HTN control interventions in HIV clinics in Nigeria. Fourth, we utilized well-established and rigorous implementation science theory, CFIR to frame potential barriers and facilitators for integrating evidence-based taskstrengthening strategies for hypertension control within HIV clinics in Nigeria.

\section{Conclusions}

This study provides important insights into barriers and facilitators for the implementation of an evidence-based intervention, task-strengthening strategies for hypertension control (TASSH), within HIV clinics in Nigeria. The findings support the importance of stakeholder engagement to foster collaboration and to examine organization readiness for intervention implementation [29]. Organizational readiness is an important factor to understand the implementation climate. Interventions that do not consider contextual and individual factors likely to facilitate or hinder intervention implementation may result in 
substandard service delivery, compromised health outcomes, and decreased public health impact [30]. An evaluation of these contextual factors will lead to increased adoption, implementation, and sustainment of evidence-based intervention and an overall improvement in public health impact. Future research should focus on identifying strategies to support the long-term practice sustainment of TASSH in Nigeria. Such approaches hold promise to improve the implementation and sustainment of intervention, as well as the quality and outcomes of care.

\section{Declarations}

\section{Ethical approval and consent to participate}

All procedures were in accordance with the ethical standards of the responsible committee on human experimentation (institutional and national) and with the Helsinki Declaration of 1975, as revised in 2000. All participants provided written informed consent and the study was approved by Nigerian Institute of Medical Research's Institutional Review Board.

\section{Consent for publication}

This manuscript does not contain any identifiable data in any form, either at the organizational level or at the individual level.

\section{Availability of data and materials}

Data is available upon request to the corresponding author

\section{Competing interests}

The authors declare that they have no competing interests

\section{Funding}

The research reported in this publication was supported by the National Heart, Lung, And Blood Institute of the National Institutes of Health under Award Number R01HL147811. The content is solely the responsibility of the authors and does not necessarily represent the official views of the National Institutes of Health.

Drs. Gbenga Ogedegbe, Juliet Iwelunmor, and Oliver Ezechi are supported by a grant received from the NIH R01 HL147811-01A1.

\section{Acknowledgments}

We would like to thank the Nigerian Institute of Medical Research and the study participants for being part of this study. 


\section{Abbreviations}

AA: Angela Aifah

AIDS: Acquired immunodeficiency syndrome

AM: Adesola Z. Musa

AR: Ashlin Rakhra

BT: Bamidele 0. Tayo

CFIR: Consolidated Framework for Implementation Research

CHEWS: Community health care workers

co: Chisom Obiezu-Umeh

CVDs: Cardiovascular diseases

DO: David Oladele

DO²: Deborah Onakomaiya

GO: Gbenga Ogedegbe

HCW: Health care worker

HIV: Human immunodeficiency viruses

HJ: Hu Jiyuan

HP: Health providers

HTN: Hypertension

II: Ifeoma Idigbe

IQR: Interquartile Range

JG: Joyce Gyamfi

Jl: Juliet Iwelunmor

LGA: Local Government Area

NCDs: Noncommunicable Diseases 
NIMR: Nigerian Institute of Medical Research

OE: Oliver Ezechi

00: Oluwatosin Odubela

PF: Practice facilitation

PHC: Primary Health Care

PLHIV: People Living with HIV

SARA: Service Availability and Readiness Assessment

SD: Standard deviations

SPSS: Statistical Package for Social Sciences

SSA: sub-Saharan Africa

TASSH: Task-strengthening strategies for hypertension

TG: Titilola Gbajabiamila

UN: Ucheoma Nwaozuru

\section{References}

1. Keates AK, Mocumbi AO, Ntsekhe M, Sliwa K, Stewart S. Cardiovascular disease in Africa: epidemiological profile and challenges. Nature Reviews Cardiology. 2017;14(5):273-93.

2. Yuyun MF, Sliwa K, Kengne AP, Mocumbi AO, Bukhman G. Cardiovascular Diseases in Sub-Saharan Africa Compared to High-Income Countries: An Epidemiological Perspective. Global heart. 2020;15(1):15-5.

3. Patel P, Speight C, Maida A, Loustalot F, Giles D, Phiri S, Gupta S, Raghunathan P. Integrating HIV and hypertension management in low-resource settings: Lessons from Malawi. PLOS Medicine. 2018;15(3):e1002523.

4. Mwagomba BLM, Ameh S, Bongomin P, Juma PA, MacKenzie RK, Kyobutungi C, Lukhele N, Mwangi KJM, Amberbir A, Klipstein-Grobusch K. Opportunities and challenges for evidence-informed HIVnoncommunicable disease integrated care policies and programs: lessons from Malawi, South Africa, Swaziland and Kenya. Aids. 2018;32:21-32.

5. Njuguna B, Vorkoper S, Patel P, Reid MJ, Vedanthan R, Pfaff C, Park PH, Fischer L, Laktabai J, Pastakia SD. Models of integration of HIV and noncommunicable disease care in sub-Saharan Africa: lessons learned and evidence gaps. AIDS. 2018;32(Suppl 1):33. 
6. Duffy M, Ojikutu B, Andrian S, Sohng E, Minior T, Hirschhorn LR. Non-communicable diseases and HIV care and treatment: models of integrated service delivery. Tropical Med Int Health. 2017;22(8):926-37.

7. Rabkin M, El-Sadr WM. Why reinvent the wheel? Leveraging the lessons of HIV scale-up to confront non-communicable diseases. Glob Public Health. 2011;6(3):247-56.

8. Rabkin M, de Pinho H, Michaels-Strasser S, Naitore D, Rawat A, Topp SM. Strengthening the health workforce to support integration of HIV and noncommunicable disease services in sub-Saharan Africa. Aids. 2018;32:47-54.

9. Mercer T, Nulu S, Vedanthan R. Innovative Implementation Strategies for Hypertension Control in Low-and Middle-Income Countries: a Narrative Review. Curr Hypertens Rep. 2020;22(5):39-9.

10. Ehrhart MG, Aarons GA, Farahnak LR. Assessing the organizational context for EBP implementation: the development and validity testing of the Implementation Climate Scale (ICS). Implementation Science. 2014;9(1):157.

11. Locke J, Beidas RS, Marcus S, Stahmer A, Aarons GA, Lyon AR, Cannuscio C, Barg F, Dorsey S, Mandell DS. A mixed methods study of individual and organizational factors that affect implementation of interventions for children with autism in public schools. Implementation Science. 2016;11(1):1-9.

12. Williams NJ, Ehrhart MG, Aarons GA, Marcus SC, Beidas RS. Linking molar organizational climate and strategic implementation climate to clinicians' use of evidence-based psychotherapy techniques: cross-sectional and lagged analyses from a 2-year observational study. Implementation Science. 2018;13(1):85.

13. Jensen-Doss A, Smith AM, Walsh LM, Ringle VM, Casline E, Patel Z, Shaw AM, Maxwell C, Hanson R, Webster R. Preaching to the Choir? Predictors of Engagement in a Community-Based Learning Collaborative. Administration Policy in Mental Health Mental Health Services Research. 2020;47(2):279-90.

14. Jacobs SR, Weiner BJ, Bunger AC. Context matters: measuring implementation climate among individuals and groups. Implementation Science. 2014;9(1):46.

15. Damschroder LJ, Aron DC, Keith RE, Kirsh SR, Alexander JA, Lowery JC. Fostering implementation of health services research findings into practice: a consolidated framework for advancing implementation science. Implementation science. 2009;4(1):50.

16. VanDevanter N, Kumar P, Nguyen N, Nguyen L, Nguyen T, Stillman F, Weiner B, Shelley D. Application of the Consolidated Framework for Implementation Research to assess factors that may influence implementation of tobacco use treatment guidelines in the Viet Nam public health care delivery system. Implementation Science. 2017;12(1):1-8.

17. Damschroder LJ, Aron DC, Keith RE, Kirsh SR, Alexander JA, Lowery JC. Fostering implementation of health services research findings into practice: a consolidated framework for advancing implementation science. Implementation science. 2009;4(1):1-15. 
18. Guba EG. ERIC/ECTJ Annual Review Paper: Criteria for Assessing the Trustworthiness of Naturalistic Inquiries. Educational Communication Technology. 1981;29(2):75-91.

19. Glisson C, Landsverk J, Schoenwald S, Kelleher K, Hoagwood KE, Mayberg S, Green P, Health RNoYM. Assessing the organizational social context (OSC) of mental health services: Implications for research and practice. Administration Policy in Mental Health Mental Health Services Research. 2008;35(1-2):98.

20. Concannon TW, Meissner P, Grunbaum JA, McElwee N, Guise J-M, Santa J, Conway PH, Daudelin D, Morrato EH, Leslie LK. A new taxonomy for stakeholder engagement in patient-centered outcomes research. J Gen Intern Med. 2012;27(8):985-91.

21. Russ SJ, Sevdalis N, Moorthy K, Mayer EK, Rout S, Caris J, Mansell J, Davies R, Vincent C, Darzi A: A qualitative evaluation of the barriers and facilitators toward implementation of the WHO surgical safety checklist across hospitals in England: lessons from the "Surgical Checklist Implementation Project". Annals of surgery 2015, 261(1):81-91.

22. Sinkowitz-Cochran RL, Burkitt KH, Cuerdon T, Harrison C, Gao S, Obrosky DS, Jain R, Fine MJ, Jernigan JA. The associations between organizational culture and knowledge, attitudes, and practices in a multicenter Veterans Affairs quality improvement initiative to prevent methicillinresistant Staphylococcus aureus. Am J Infect Control. 2012;40(2):138-43.

23. Waltz TJ, Powell BJ, Fernández ME, Abadie B, Damschroder LJ. Choosing implementation strategies to address contextual barriers: diversity in recommendations and future directions. Implementation Science. 2019;14(1):42.

24. Green AE, Aarons GA. A comparison of policy and direct practice stakeholder perceptions of factors affecting evidence-based practice implementation using concept mapping. Implementation Science. 2011;6(1):104.

25. Powell BJ, Mandell DS, Hadley TR, Rubin RM, Evans AC, Hurford MO, Beidas RS. Are general and strategic measures of organizational context and leadership associated with knowledge and attitudes toward evidence-based practices in public behavioral health settings? A cross-sectional observational study. Implementation Science. 2017;12(1):1-13.

26. Aarons GA, Green AE, Trott E, Willging CE, Torres EM, Ehrhart MG, Roesch SC. The roles of system and organizational leadership in system-wide evidence-based intervention sustainment: a mixedmethod study. Administration Policy in Mental Health Mental Health Services Research. 2016;43(6):991-1008.

27. Palinkas LA, Aarons GA, Horwitz S, Chamberlain P, Hurlburt M, Landsverk J. Mixed method designs in implementation research. Administration Policy in Mental Health Mental Health Services Research. 2011;38(1):44-53.

28. Green CA, Duan N, Gibbons RD, Hoagwood KE, Palinkas LA, Wisdom JP. Approaches to mixed methods dissemination and implementation research: methods, strengths, caveats, and opportunities. Administration Policy in Mental Health Mental Health Services Research. 2015;42(5):508-23. 
29. Scaccia JP, Cook BS, Lamont A, Wandersman A, Castellow J, Katz J, Beidas RS. A practical implementation science heuristic for organizational readiness: $R=M C 2$. Journal of Community Psychology. 2015;43(4):484-501.

30. Aarons GA, Ehrhart MG, Farahnak LR, Hurlburt MS. Leadership and organizational change for implementation (LOCI): a randomized mixed method pilot study of a leadership and organization development intervention for evidence-based practice implementation. Implementation Science. 2015;10(1):11.

\section{Tables}

Table 1. Assessment of Implementation Climate for implementing TASSH within HIV clinics $(n=29)$ in Lagos, Nigeria

\begin{tabular}{|lllll|}
\hline Implementation Climate Subscales & No. of items & Cronbach's alpha & Mean & SD \\
\hline Focus on TASSH & 3 & 0.93 & 1.77 & 0.59 \\
\hline Educational Support for TASSH & 3 & 0.91 & 1.31 & 0.68 \\
\hline Recognition for TASSH & 3 & 0.84 & 1.14 & 0.79 \\
\hline Rewards for TASSH & 2 & 0.96 & 0.73 & 0.48 \\
\hline Selection for Openness to TASSH & 3 & 0.88 & 1.15 & 0.73 \\
\hline Total & 14 & 0.89 & 1.23 & 0.46 \\
\hline
\end{tabular}


Table 2: Identified facilitators and barriers based on CFIR domains and constructs

CFIR Domain Barriers/Facilitators CFIR Construct Description

Intervention Facilitators

Characteristics

Relative

Advantage of

- Reduce the workloads of

TASSH

overburdened workers

- Improve overall efficiency

- The ability for patients to access care

in the same clinic or location

- Reduce clinic wait times

- Reduce the stigma faced by PLWHIV

\begin{tabular}{|c|c|c|c|}
\hline & Barriers & $\begin{array}{l}\text { Complexity of } \\
\text { TASSH }\end{array}$ & $\begin{array}{l}\text { Potential for disagreements and } \\
\text { conflicts over roles and role boundaries }\end{array}$ \\
\hline \multirow[t]{4}{*}{ Inner Setting } & \multirow[t]{2}{*}{ Facilitators } & $\begin{array}{l}\text { Compatibility } \\
\text { with existing } \\
\text { workflows and } \\
\text { processes }\end{array}$ & $\begin{array}{l}\text { Integration of the existing CHEWs into } \\
\text { the national structures to improve } \\
\text { referral systems }\end{array}$ \\
\hline & & $\begin{array}{l}\text { Access to } \\
\text { knowledge and } \\
\text { information }\end{array}$ & $\begin{array}{l}\text { Availability of educational support for } \\
\text { evidence-based practice for } \\
\text { hypertension management within the } \\
\text { HIV clinics }\end{array}$ \\
\hline & \multirow[t]{2}{*}{ Barriers } & $\begin{array}{l}\text { Unavailable } \\
\text { resources and } \\
\text { support }\end{array}$ & $\begin{array}{l}\text { - Inadequate availability of diagnostic } \\
\text { equipment and drugs for HTN across } \\
\text { HIV clinics }\end{array}$ \\
\hline & & $\begin{array}{l}\text { Networks and } \\
\text { communication }\end{array}$ & $\begin{array}{l}\text { Weak referral networks and patient } \\
\text { tracking mechanism for HTN } \\
\text { management within HIV clinics }\end{array}$ \\
\hline
\end{tabular}

Outer setting Facilitators

Needs and resources of

- Supports patients' needs those served by the intervention

Incentives

- Provision of non-monetary incentives in the form of professional development

Barriers

External

- Existing national polices on NCD

Policies management and Task-sharing are not implemented in the clinics

- No specific action in task-shifting policy to minimize workload

Characteristics Facilitators of individuals
Knowledge and beliefs
- Knowledge of benefit for implementing TASSH and values placed on the importance of EBP

Implementation Facilitators Planning

- Accommodate shift preferences and Process 
nurses; Implement simplified data collection tools; Align program goals with national guidelines for NCDs

\section{Engagement}

- Identify program champions and change agents within the organization; Consistent leadership support; Increase community awareness of TASSH

\section{Figures}

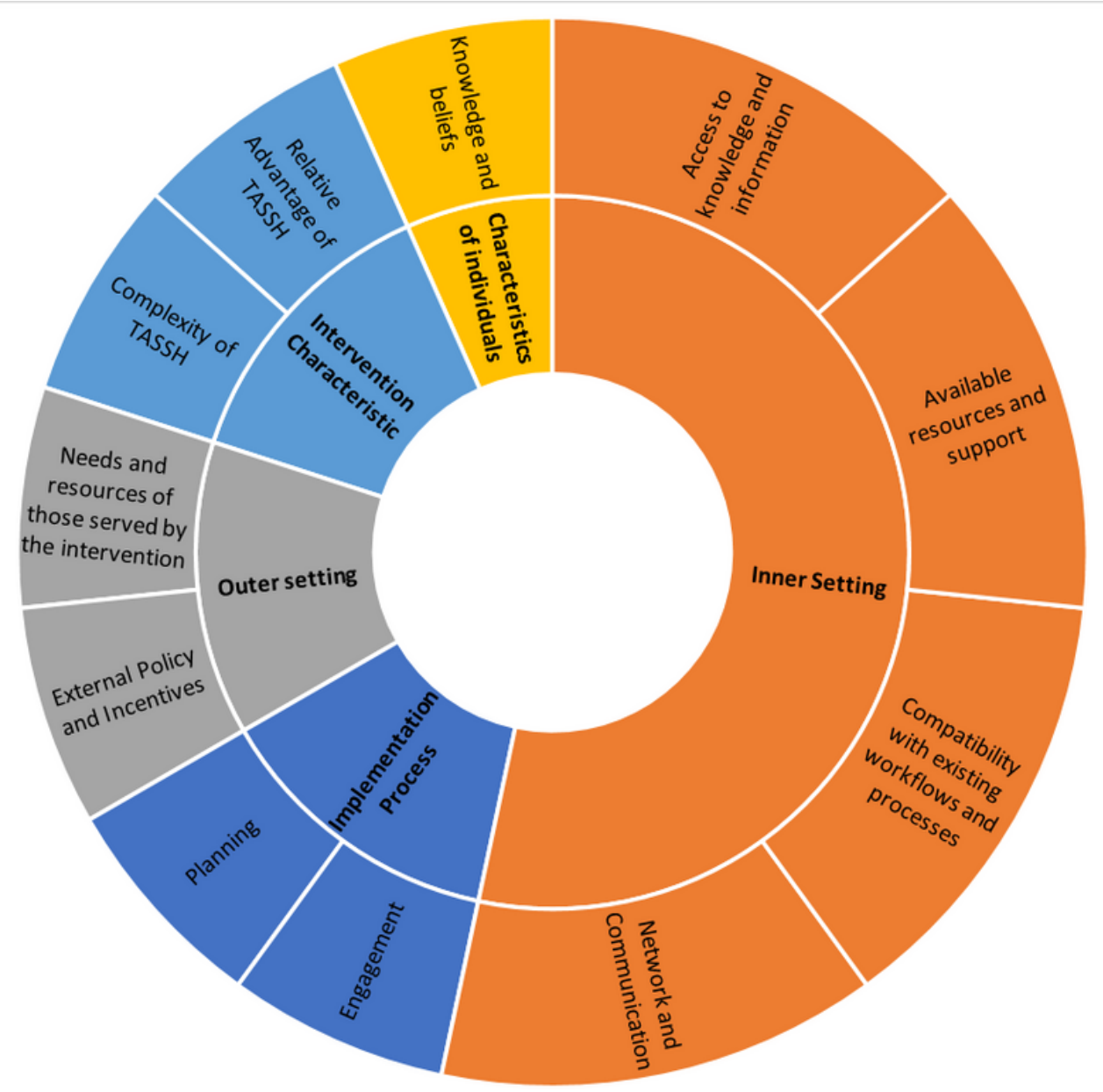

Figure 1

Overview of main influential factors ordered by CFIR domain and constructs

\section{Supplementary Files}

This is a list of supplementary files associated with this preprint. Click to download.

- STROBE.docx 